\title{
Tenencia de tierra y aguas de pequeños y medianos agricultores del valle de Azapa, Arica, Chile
}

\author{
Ownership of land and water in small and medium farmers of Azapa valley \\ Camila Aguilar, Christopher Röper, Pilar Mazuela ${ }^{1 *}$, Amador Torres ${ }^{2}$
}

\begin{abstract}
RESUMEN
El valle de Azapa, uno de los valles costeros de la XV Región de Arica y Parinacota, en el extremo norte de Chile, presenta condiciones climáticas excepcionales para el cultivo de hortalizas durante todo el año, siendo el principal proveedor de hortalizas de la zona central durante el invierno. El alto costo de la tierra y escasa disponibilidad de agua ha incentivado las mejoras tecnológicas en los procesos de producción para mantener esta actividad sustentable en el tiempo. El objetivo de este trabajo fue describir la tenencia de tierra por tamaño de explotación y el número de acciones de agua asociados a la explotación de pequeños y medianos agricultores del valle de Azapa. Para ello se realizó una encuesta en una muestra correspondiente al 5\% de los agricultores del valle de Azapa. Los resultados indican que el $27 \%$ de los agricultores encuestados tiene menos de una acción de agua por ha; 35\% tiene más de una acción de agua por ha y el $38 \%$ tiene una acción de agua por ha.
\end{abstract}

Palabras clave: acciones de agua, PYME, explotación, primor

\begin{abstract}
Azapa is one of the coastal valleys of Arica and Parinacota region, in the extreme north of Chile, that presents exceptional climatic conditions for the cultivation of vegetables off season, being the main supplier of vegetables in the Central area during the winter. The high cost of land and little water availability has encouraged technological improvements in production processes to maintain this sustainable activity over time. The aim of this study was to describe the land tenure by farm size and the number of shares of water associated with the exploitation of small and medium farmers in Azapa Valley. For this, a survey was conducted on a sample corresponding to the 5\% of farmers of Azapa valley. The results indicate that $27 \%$ of farmers surveyed have less of an action of water per hectare; 35\% have more than one share of water per hectare and $38 \%$ have an action of water per hectare.

Key words: shares of water, SMEs, farm, off-season
\end{abstract}

\section{Introducción}

La actividad agrícola en la Región de Arica y Parinacota se desarrolla principalmente en los valles costeros destacando el valle de Azapa por superficie cultivada, intensificación cultural, diversificación de cultivos y cercanía a la ciudad. Espina (1971) en el estudio agroeconómico solicitado por la Junta de Adelanto de Arica (JAA) determinó la producción, organización, manejo y comercialización de la agricultura del valle de Azapa. Los principales resultados de este estudio demuestran que la situación del valle no ha variado mucho en los últimos 43 años respecto del manejo cultural que impide desarrollar todo el potencial productivo de recursos como la tierra y el agua. La productividad ha mejorado significativamente cuadruplicando los rendimientos medios en torno a $30 \mathrm{t} \mathrm{ha}^{-1}$ de principios de los años 70. Sin embargo, la introducción de sistemas de riego más eficientes, pasando del riego por caracol a riego por goteo, ha sido insuficiente para mejorar la eficiencia hídrica y la sustentabilidad del recurso hídrico. Esto debido a que las tecnologías que se adoptan son transferidas tal como en el lugar de origen, sin considerar que las condiciones de clima, agua y suelo son diferentes.

No solo es importante la incorporación de nueva tecnología en agricultura para hacer más eficiente el uso de recursos como la tierra y el agua, además, es necesario capacitar a los agricultores en técnicas de manejo que permitan optimizar el uso de estos recursos. En el valle de Azapa, el riego mecanizado,

\footnotetext{
1 Departamento de Producción Agrícola, Universidad de Tarapacá, Arica, Chile.

2 Departamento de Recursos Ambientales, Universidad de Tarapacá, Arica, Chile.

* Autor correspondiente: pmazuela@uta.cl
}

Fecha de Recepción: 1 Agosto, 2015.

Fecha de Aceptación: 1 Septiembre, 2015. 
especialmente el sistema por goteo se ha masificado entre los agricultores a contar de los años 90 . Según el último censo agropecuario (INE, 2008), la región de Arica y Parinacota cuenta con una superficie de 2012,63 hectáreas regadas con el sistema de riego por goteo, cifra que se concentra principalmente en el valle de Azapa. Otra manera de mejorar la eficiencia de tierra y agua es la incorporación de sistemas de protección que permiten aumentar la productividad por superficie cultivada y mejorar la eficiencia hídrica. En la última década se observa una tendencia a mejorar los procesos de producción donde más de 500 hectáreas de cultivo están bajo malla (Riquelme et al., 2013). La eficiencia del uso del agua en cultivos de tomate al aire libre en el valle de Azapa alcanza a 12,5 $\mathrm{kg}$ producidos por $\mathrm{m}^{3}$ de agua aporta, cifra que se duplica en sistemas de producción bajo protección (González et al., 2013). En el mismo estudio se observó que los rendimientos medios de tomate al aire libre y bajo malla alcanzan 7,6 y $20,6 \mathrm{~kg} \mathrm{~m}^{-2}$.

\section{Agua}

Espina (1971) indica que el agua que dispone Arica y el valle de Azapa proviene de la precordillera al oriente de la sierra de Huaylillas y llega por el canal Lauca o por aguas subterráneas. Sin embargo, es el río San José, o Azapa, el principal recurso hidrológico de este valle cuyas aguas provienen de las precipitaciones de las quebradas de Seca, Chusmiza y Ticnamar. Las precipitaciones se concentran entre diciembre y marzo y se caracterizan por ser de gran intensidad y corta duración, originando un aumento de los caudales que provocan un régimen de escurrimiento con características de avenida (Klohn, 1972). El agua de este río corre superficialmente durante los años lluviosos y subterráneamente en los años secos, apareciendo por vertientes, norias o pozos. Desde las fuentes primarias del agua, los sedimentos aluviales nacientes que conforman el lecho del valle de Azapa tienen mayor potencialidad como fuente de agua subterránea, se ubican entre 0 y $70 \mathrm{~m}$ de profundidad, dando lugar a napas libres y algunas semiconfinadas (Karsulovic, 1968; Jorquera, 1971; Edwards y Karzulovic, 1981).

Según antecedentes de la Comunidad de Aguas Canal Azapa ingresa al canal Azapa en bocatoma un caudal promedio de $0,705 \mathrm{~m}^{3} \mathrm{~s}^{-1}$, siendo actualmente la principal fuente de agua para la agricultura del valle de Azapa. El agua subterránea es extraída mediante bombas desde sondajes y norias, como también hasta hace algunos años por gravedad desde los afloramientos naturales (vertientes) existentes en el valle de Azapa, las que hoy se encuentran sin escurrimiento por la disminución de las precipitaciones en la cabecera de la cuenca y por el aumento de la extracción desde los sondajes y norias. La sobreexplotación del acuífero del San José está afectando la disponibilidad y calidad de agua debido a: 1) habilitación de suelos para el cultivo de hortalizas; 2) ineficiencia en las técnicas de riego y 3 ) desequilibrios nutricionales en las plantas por técnicas deficientes en la fertilización del cultivo. El acuífero del río San José, además, es la fuente de agua potable para la ciudad de Arica. Según la Dirección General de Aguas (DGA, 2008), la explotación del acuífero de Azapa está sobre su capacidad sustentable, del orden de $7001 \mathrm{seg} .^{-1}$, pues los derechos de aprovechamiento de agua superan los $30001 \mathrm{seg} .{ }^{-1}$ y la explotación real es de 10001 seg. ${ }^{-1}$.

\section{Suelos}

El ancho del valle es variable y fluctúa entre 700 y $2.200 \mathrm{~m}$; con una pendiente de $1,7 \%$ y un largo aproximado de $58 \mathrm{~km}$. Los suelos son aluviales con una marcada estratificación. Las texturas más comunes: en la superficie, la franco arcillosa fina a franca y, en profundidad, se alternan las estratas de texturas arenosas y, ocasionalmente, arcillosas. Los suelos poseen una buena saturación de bases, son calcáreos, de reacción moderadamente alcalina y de buena porosidad. Sin embargo, son de poca profundidad y con un exceso de pedregosidad.

Debido a los procesos depositacionales, los suelos del valle de Azapa tienen una estratificación muy marcada. Meléndez y Wright (1961) describen la parte baja del valle con estratos gruesos y poca fluctuación de textura; en la parte media a baja se observan suelos aluviales profundos; en tanto, el sector medio superior tiene suelos profundos, de texturas medias, color pardo a pardo oscuros, estructura de bloques subangulares, friables y de buena permeabilidad. Poco más del 19\% de la superficie del valle son suelos que ocupa la caja del río San José, con abundancia de piedras, muy delgados, de color pardo, textura gruesa, sin estructura y de permeabilidad muy rápida. IRENCORFO (1976) indica que el $62 \%$ de los suelos del 
valle de Azapa corresponden a suelos II, III y IV, según las Clases de Capacidad de Uso y un 30,7\% corresponden a clase VII. En las últimas décadas se ha habilitado superficie cultivable, bien por relleno y acondicionamiento de zonas pedregosas en la caja del río San José o por habilitación de laderas de los cerros aledaños.

\section{Clima}

El clima es la principal ventaja de Arica para producir hortalizas al aire libre para consumo fresco durante todo el año. Predominan las condiciones de clima de desierto costero con nubosidad abundante, ausencia de heladas, vientos moderados, alta humedad relativa y alta radiación solar directa, durante todo el año. Al comparar las temperaturas medias de principios de la década de los 70 (Espina, 1971) y las indicadas por Torres y Acevedo (2008), se observa que la temperatura media anual ha bajado, principalmente por una disminución en las temperaturas mínimas medias. Sin embargo, las máximas medias han aumentado, lo que origina una mayor fluctuación entre temperaturas mínimas y máximas medias pasando de $6,6{ }^{\circ} \mathrm{C}$ a $9,8{ }^{\circ} \mathrm{C}$ (González et al., 2013).

El objetivo de este trabajo fue describir la tenencia de tierra por tamaño de explotación y el número de acciones de agua asociados a la explotación de pequeños y medianos agricultores del valle de Azapa.

\section{Metodología}

Se realizó una encuesta utilizada para el levantamiento en terreno que se estructuró tomando en cuenta los antecedentes personales del encuestado, antecedentes generales del predio, antecedentes del sistema de riego y antecedentes complementarios (Figura 1).

\section{Localización y descripción de los predios}

La encuesta se realizó a 48 agricultores de la parte media y alta del valle de Azapa, en los sectores de Pan de Azúcar, Cabuza, La Cruz y Cerro Blanco, ubicados entre los kilómetros 13 y 45 del valle. En estos sectores se concentran los pequeños y medianos agricultores, pues en la parte baja del pueblo de San Miguel, gran parte de los predios tiene uso habitacional.

\section{Periodo descrito}

Los datos corresponden a la encuesta aplicada en el periodo comprendido entre mayo y octubre de 2014.

\section{Muestreo}

La muestra fue tomada al azar considerando el $5 \%$ del total de informantes que tiene riego por goteo según el INE (2008). Los datos fueron tabulados en una planilla Excel para el análisis descriptivo de la relación tierra/acciones de agua.

\section{Resultados}

Los resultados de superficie por tamaño de explotación, número de acciones de agua por explotación y relación número de acciones de agua por unidad de superficie se describen en la Tabla 1. Como se observa, casi el 39\% de la superficie cuenta con una acción de agua, correspondiente al $42,4 \%$ del total de acciones de agua. Sin embargo, la mayor superficie encuestada $(42,2 \%)$ cuenta con una media de 0,5 acción de agua, lo que sugiere una alta demanda por el recurso hídrico entre pequeños y medianos agricultores. En la Figura 2 se observa que la mayoría de los pequeños y medianos agricultores (38\%) tiene una acción de agua por ha y el 27\% tiene menos de una acción de agua por ha. Al analizar la media de acción de agua de agricultores con un número de acciones de agua mayor a 1, esta alcanza a 1,8, como se observa en la Tabla 1 , y corresponde al $35 \%$ de los agricultores.

Al analizar el porcentaje de pequeños y medianos agricultores según tamaño de la explotación (Figura 3), se observa que el 54\% de estos cuentan con explotaciones de superficie entre 0,5 y 2 ha. Al comparar la evolución de superficie de las explotaciones según el INE $(1998,2008)$, se observa que el porcentaje de pequeños y medianos agricultores con explotaciones menores a 0,5 ha ha aumentado de $28 \%$ a $33 \%$ desde 1997 a 2015. Esto se puede atribuir a una mayor subdivisión de las explotaciones con objetivos habitacionales. Los agricultores con superficies mayores a 5 ha han disminuido del $24 \%$; $20 \%$ y $15 \%$, según los censos de 1997, 2007 y resultados de esta encuesta. Finalmente, se debe destacar que las explotaciones con superficies entre 1 y 5 ha han duplicado el porcentaje; de $27,5 \%$ en 1997 a $52 \%$ en 2007 , 


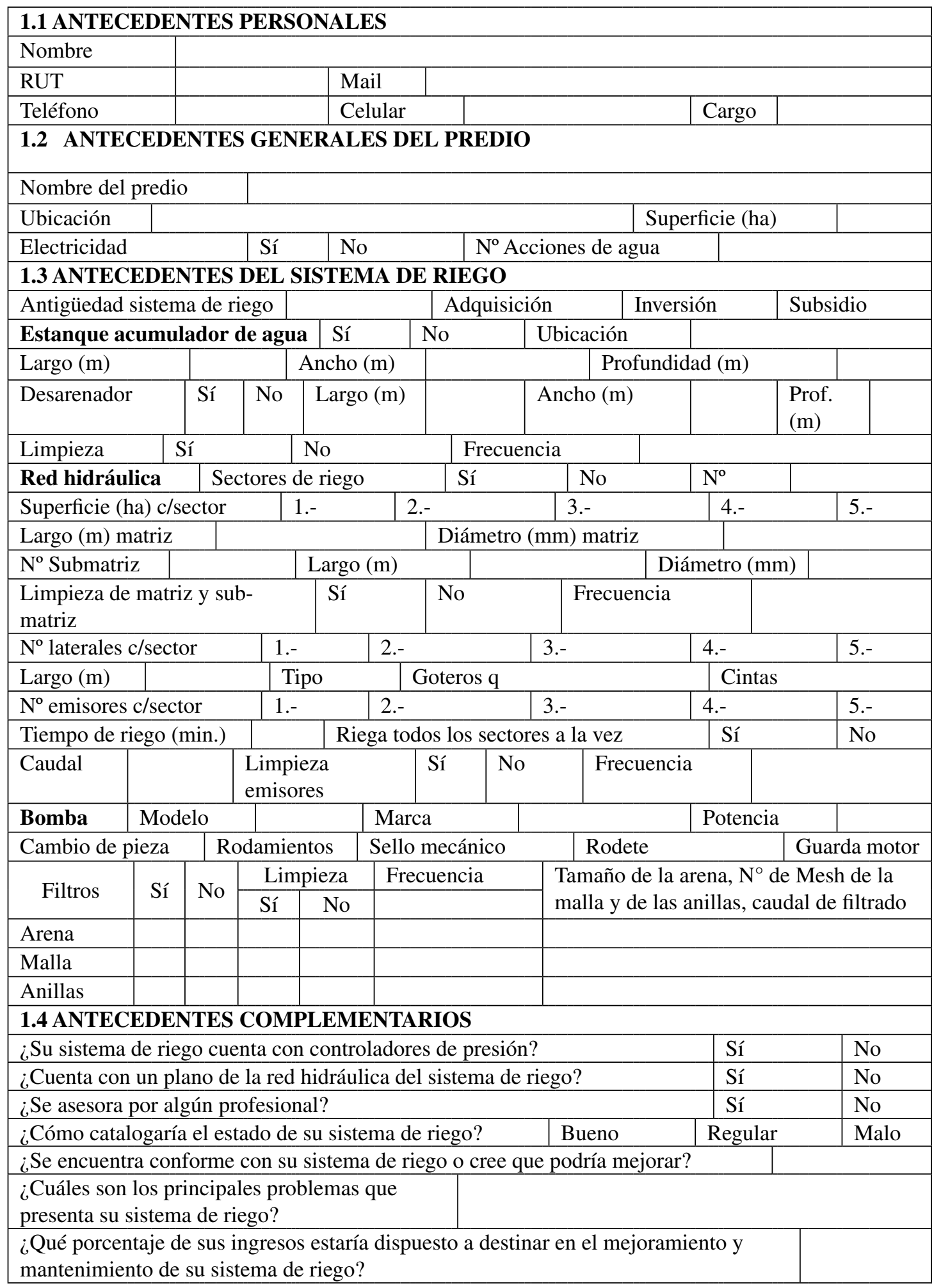

Figura 1. Encuesta para el levantamiento de información. 
Tabla 1. Tamaño de explotación, por superficie (ha) y porcentaje (\%); acciones de agua por explotación, por número $\left(\mathrm{N}^{\circ}\right)$ y porcentaje (\%) y relación número de acciones de agua por unidad de superficie (AA/ha) de pequeños y medianos agricultores del valle de Azapa.

\begin{tabular}{|c|c|c|c|c|c|}
\hline & \multicolumn{2}{|c|}{ Superficie } & \multicolumn{2}{|c|}{$\mathrm{N}^{\circ}$ acciones de agua (AA) } & \multirow{2}{*}{$\frac{\text { Media }}{\text { AA/ha }}$} \\
\hline & ha & $\%$ & $\mathrm{~N}^{\circ}$ & $\%$ & \\
\hline $\mathrm{AA}>\mathrm{ha}$ & 27,5 & 18,9 & 48,7 & 36,6 & 1,8 \\
\hline $\mathrm{AA}=\mathrm{ha}$ & 56,5 & 38,9 & 56,5 & 42,4 & 1,0 \\
\hline $\mathrm{AA}<\mathrm{ha}$ & 61,4 & 42,2 & 28,0 & 21,0 & 0,5 \\
\hline Total & 145,4 & & 133,2 & & 0,9 \\
\hline
\end{tabular}

AA: acciones de agua; ha: hectárea

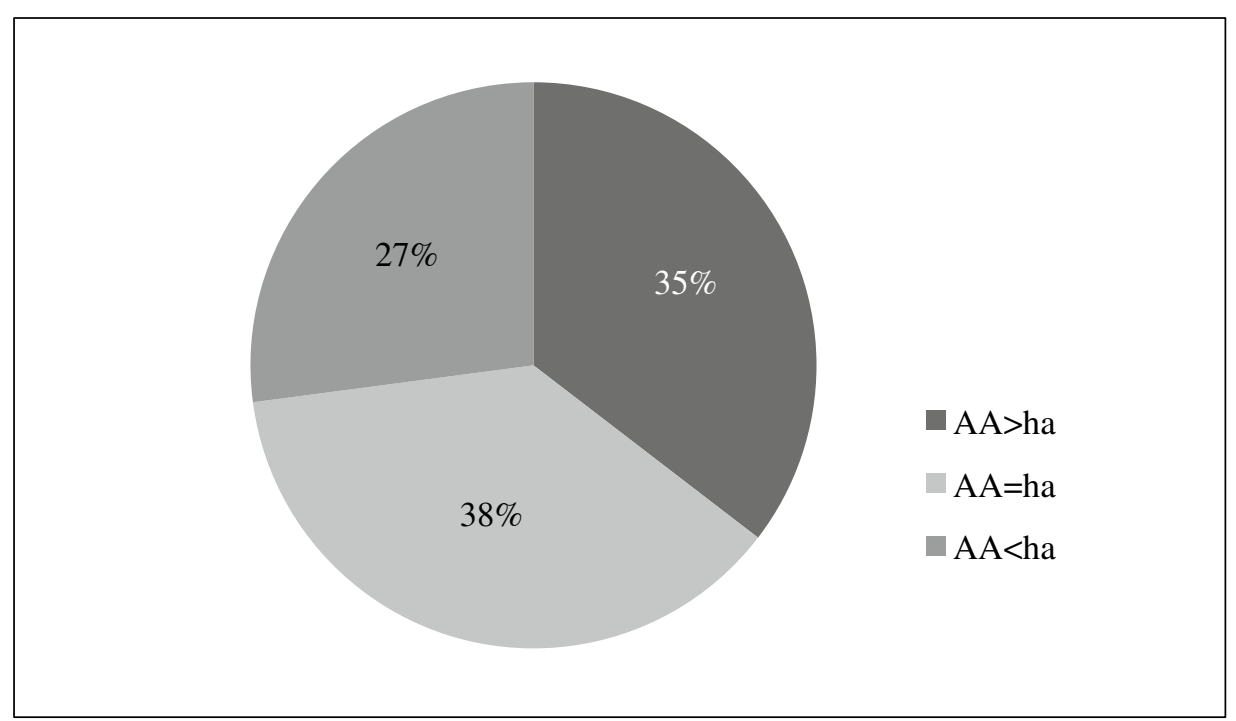

Figura 2. Porcentaje (\%) de pequeños y medianos agricultores con relación a tenencia acción de agua/superficie (AA/ha): AA $>$ ha, $\mathrm{AA}=\mathrm{ha}, \mathrm{AA}<\mathrm{ha}$.

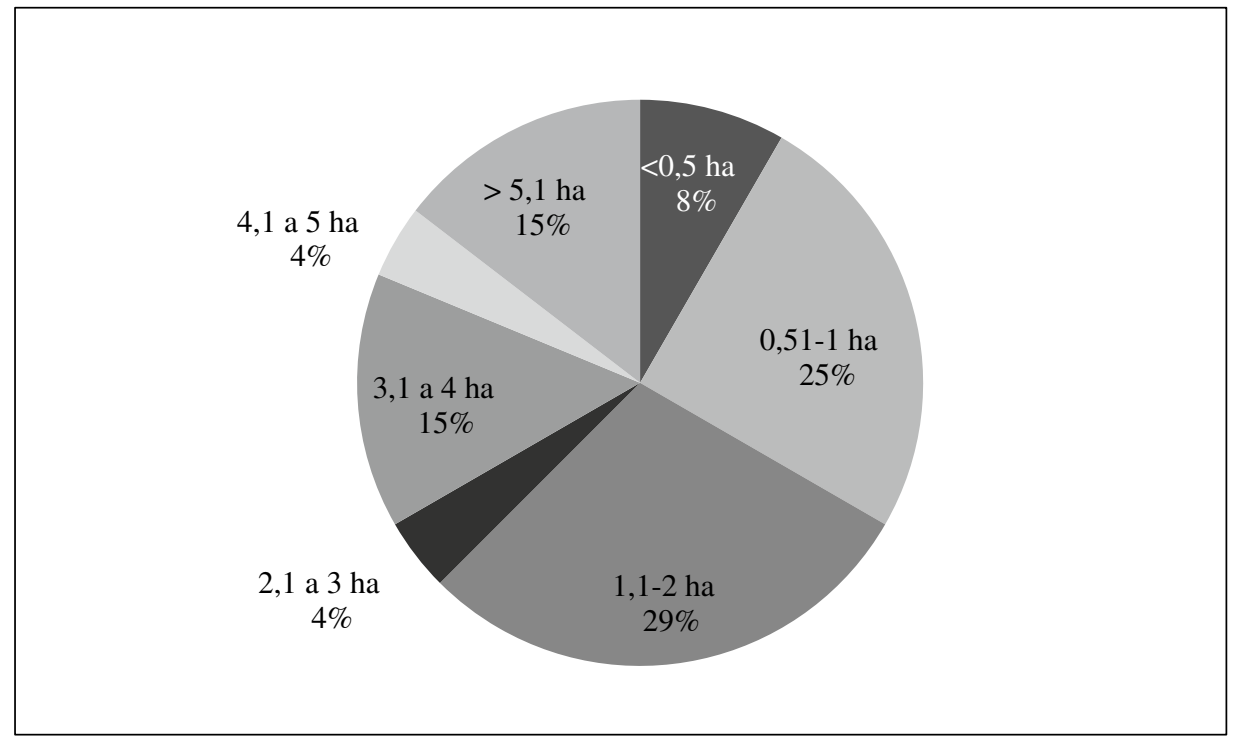

Figura 3. Porcentaje de pequeños y medianos agricultores, según tamaño de la explotación (ha). 
porcentaje que se mantiene, según los resultados de esta encuesta.

\section{Conclusión}

Los resultados de este trabajo sugieren que la mayoría de las explotaciones tiene tamaño entre 1 y 5 ha, donde el porcentaje de pequeños y medianos agricultores alcanza un 52\% del total de agricultores. No se observa una tendencia a concentra la superficie agrícola, por el contrario, las explotaciones menores a 0,5 ha han aumentado, alcanzando al $8 \%$ del total. Se observa que la mayoría de los agricultores (38\%) tiene una relación de una acción de agua por hectárea, con una tendencia a disminuir la proporción de la acción de agua por superficie. Esto sugiere que el agricultor tiende a aumentar la eficiencia en el uso de agua. El 35\% de los agricultores tiene una media de 1,8 acción de agua por unidad de superficie, lo que podría sugerir que la venta de terrenos agrícolas no va asociada a la venta de agua.

\section{Agradecimientos}

Este trabajo ha sido financiado por el proyecto Estudiantil UTA 9721-14.

\section{Literatura Citada}

Dirección General de Aguas.

2008. Las Mesas de Agua y las experiencias recientes del MOP en el ámbito de la gestión del agua. DGA, Región de Arica y Parinacota. 9 p.

Edwards, G.; Karzulovic, K.

1981. Febrero. "Plan Maestro de Acción Inmediata para el Sistema de Riego del Valle de Azapa”. Tomo II. Informe General. Región de Tarapacá. Ministerio de Obras Públicas, Dirección de Riego. Chile.

Espina, L.

1971. Estudio agroeconómico del valle de Azapa. Junta de Adelanto de Arica-Universidad de Chile, Santiago de Chile. $155 \mathrm{p}$.

González, F.; Riquelme, A.; Contreras, P.; Mazuela, P.

2013. Antecedentes generals para la sustentabilidad de la producción horticola en el valle de Azapa, Arica, Chile. Idesia, 31(4): 119-123

IREN-CORFO.

1976. Inventario de Recursos Naturales por métodos de percepción del satélite LANDSAT. I Región de Tarapacá. Convenio IREN-SERPLAC. Santiago, Chile, pp. 50-110.

Instituto Nacional de Estadísticas.

2010. Información Hortícola. Publicación Especial 2008-2009.

INE Ediciones, Santiago de Chile, 128 p.

Instituto Nacional de Estadísticas.

1999. VI Censo Nacional Agropecuario, Región de Tarapacá. Edición Rossana Espinosa Peralta. Iquique, Chile. 473 p.
Instituto Nacional de Estadísticas.

2008. VII Censo Agropecuario y Forestal 2006-2007. Resultados preliminares. INE Ediciones, Santiago de Chile, $444 \mathrm{p}$.

Jorquera, L.

1971. Recursos de agua y su aprovechamiento en el Valle de Azapa. Mimeografiado. 32 p.

Karsulovic, J.

1968. Estudio hidrogeológico de la Región de Arica. Santiago, Universidad de Chile, pp. 26-38.

Klohn,W.

1972. Hidrografía de las zonas desérticas de Chile, ed. Jean B., Proyecto CHI-35 Investigación de los recursos hidráulicos en el Norte Grande PNUD para el desarrollo, Santiago, Chile. 188 p.

Meléndez, E. y Wright, Ch.

1961. Estudio de los suelos del Valle de Azapa. Ministerio de Agricultura, $45 \mathrm{p}$.

Riquelme, A.; González, F.; Contreras, P.; Mazuela, P.

2013. Manejo del cultivo de hortalizas y su efecto en la sustentabilidad de un valle costero del desierto de Atacama, Chile. Idesia, 31 (3): 113-117.

Torres, A. y Acevedo, E.

2008. El problema de salinidad en los recursos suelo y agua que afectan el riego en los valles de Lluta y Azapa en el norte de Chile. Idesia, 26 (3): 31-44. 